\title{
Research On Frequency-Domain Kernel Identification Of Volterra Series
}

\author{
Shihao Wu, Yafeng Meng ${ }^{*}$, Bo Wang \\ Department of Electronics and Optics Engineering, Machanical Engineering College \\ Shijiazhuang 050003, China \\ "Corresponding author: Yafeng Meng, E-mail addresses: radarm@163.com
}

Key words: Volterra series; non-linear; frequency-domain kernel; identification method

\begin{abstract}
In this paper, the identification method of Volterra series frequency-domain kernel is introduced from two aspects: parameter identification and non-parameter identification, then gives prospect. Volterra series theory has been applied and developed in recent years, becomes one of the most effective tools for nonlinear system analysis and modeling. The kernel of Volterra series can reflect the fault characteristics well. Therefore the problem of kernel identification has been the focus of research. Among them, the frequency-domain kernel identification is concerned because of many advantages, such as strong robustness, no need to determine the memory length and so on.
\end{abstract}

\section{Introduction}

In the real world, most of the subjects to be researched are non-linear. There are many nonlinear analysis methods proposed by scholars, Volterra series is one of the powerful tools. As the promotion of Taylor series, Volterra series was proposed by mathematician Vito Volterra in 1880 , Wiener, the founder of American Cybernetics, first applied it to the analysis of non-linear systems in 1942. At present, it has been widely used in electronic engineering [1], mechanical equipment [2], control engineering [3] and other fields. Volterra series can be arbitrary approximated by a continuous function in theory, which can represent a large class of non-linear systems [4]. The key to modeling the system by using Volterra series is to identify the kernel function. It extends the convolution of linear system to a series of multidimensional convolution, which can describe the essential characteristic of non-linear system, has a distinct physical meaning. The kernel function of Volterra series is divided into time-domain kernel and frequency-domain kernel (or generalized frequency response function, GFRF). Due to the disadvantage of the time-domain can only reflect the transient process, need to specify the memory length, Only for stationary random signal and so on, instead, GFRF is more intuitive and accurate to reflect non-linear system, with the characteristics of no restrictions of the excitation signal, no need to determine the memory length and strong robustness. So GFRF, based on Volterra series model, is widely used in the study of non-linear system. In this paper, the identification method of GFRF is introduced by the parameter identification method and Nonparametric identification method.

\section{Basic knowledge of Volterra series}

To facilitate the understanding of the content to be introduced next, the basic theory of Volterra series is briefly described [5].

The input-output relationship of non-linear analog circuit system is complex, Volterra series provides a solution similar to the impulse response function in linear systems.

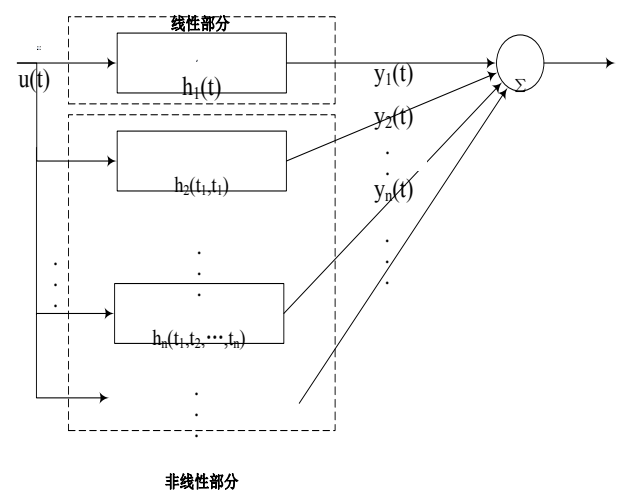

Figure 1: The relationship of non-linear system between the input and output based on Volterra series

Any continuous time invariant weakly non-linear dynamic system, can be fully described by Volterra series. As shown in Figure 1, non-linear system can be described as a sum of two parts: linear and non-linear.

$$
y(\mathrm{t})=\sum_{n=0}^{\infty} y_{n}(\mathrm{t})
$$


Where

$$
y_{n}(\mathrm{t})=\int_{-\infty}^{\infty} \ldots \int_{-\infty}^{\infty} h_{n}\left(\tau_{1}, \tau_{2}, \ldots \tau_{n}\right) \prod_{i=1}^{n} u\left(\mathrm{t}-\tau_{i}\right) d \tau_{i}
$$

$y_{n}(\mathrm{t})$ is the output response of the system, $y_{1}(\mathrm{t})$ corresponds to linear partial output of the system, $y_{n}(\mathrm{t})(n \geq 2)$ corresponds to non-linear partial output of the system, $h_{n}\left(\tau_{1}, \tau_{2}, \ldots \tau_{n}\right)$ is the $\mathrm{n}$ th-order Volterra time-domain kernel, The Fourier transform of Eq.(1.1) can be obtained:

$$
Y(\mathrm{j} \omega)=\sum_{n=1}^{\infty} Y_{n}(\mathrm{j} \omega)
$$

Where

$$
\begin{aligned}
& Y_{n}(\mathrm{j} \omega)=\frac{1}{(2 \pi)^{n-1}} \int_{-\infty}^{\infty} \ldots \int_{-\infty}^{\infty} H_{n}\left(\omega-\omega_{2}-\ldots-\omega_{n}, \omega_{2}, \ldots, \omega_{n}\right) \\
& \times u\left(\omega-\omega_{2}-\ldots-\omega_{n}, \omega_{2}, \ldots, \omega_{n}\right) \mathrm{u}\left(\omega_{2}\right) \ldots \mathrm{u}\left(\omega_{n}\right) \mathrm{d} \omega_{2} \ldots d \omega_{n}
\end{aligned}
$$

$$
\begin{aligned}
& H_{n}\left(\omega_{1}, \omega_{2}, \ldots, \omega_{n}\right)=\int_{-\infty}^{\infty} \ldots \int_{-\infty}^{\infty} h_{n}\left(\tau_{1}, \tau_{2}, \ldots \tau_{n}\right) \\
& \times \mathrm{e}^{-j\left(\omega_{1} \tau_{1}+\omega_{2} \tau_{2}+\ldots+\omega_{n} \tau_{n}\right)} d \tau_{1} d \tau_{2} \ldots d \tau_{n}
\end{aligned}
$$$$
\text { In } \quad \omega=\omega_{1}+\omega_{2}+\omega_{3}+\ldots+\omega_{n}
$$

$H_{n}\left(\omega_{1}, \omega_{2}, \ldots, \omega_{n}\right)$ is multidimensional Fourier transform of $h_{n}\left(\tau_{1}, \tau_{2}, \ldots \tau_{n}\right)$, As shown in Eq. (1.5), $H_{n}\left(\omega_{1}, \omega_{2}, \ldots, \omega_{n}\right)$ is the GFRF of nonlinear system.

In the analysis of non-linear systems based on Volterra series, GFRF does not change with the input signal of the system, Only relevant to the characteristic parameters of the system. People can understand many non-linear phenomena intuitively and accurately. It has high reliability. The identification method is divided into parameter identification method and nonparametric identification method.

\section{Parameter identification method}

Parameter identification solves the kernel function by decomposing the correlation term and iteration, represented by a specific analytical formula. The parameter identification method of GFRF can be divided into the differential equation model, the non-linear autoregressive model and the non-linear time delay model according to the continuous time, discrete and time delay [6].
In the continuous time non-linear systems, autoregressive model is described as:

$$
\sum_{m=1}^{M} \sum_{p=0}^{m} \sum_{l_{1}, l_{p+q=0}}^{L} c_{p, q}\left(l_{1}, \ldots, l_{p+q}\right) \prod_{i=1}^{p} D^{l_{i}} y(t) \prod_{i=p+1}^{p+q} D^{l_{i}} u(t)=0
$$

Which $D$ represents the differential operator, $l_{i}$ represents the order of the differential, $c_{p, q}(\cdot)$ is the coefficient of the model.

The autoregressive model of the non-linear discrete-time system is described as:

$$
y(t)=\sum_{m=1}^{M} \sum_{p=0}^{m} \sum_{k_{1}, k_{p+q=0}}^{K} c_{p, q}\left(k_{1}, \ldots, k_{p+q}\right) \prod_{i=1}^{p} y\left(t-k_{i}\right) \prod_{i=p+1}^{p+q} u\left(t-k_{i}\right)
$$

Which $t$ represents the sample time serial number, $k_{i}$ is the discrete time delay.

The non-linear time delay model of continuous non-linear system is described as:

$$
\begin{aligned}
& \sum_{m=1}^{M} \sum_{p=0}^{m} \sum_{l_{1}, l_{p+q=0}}^{L} \sum_{k_{1}, k_{p+q}=0}^{K} c_{p, q}\left(l_{1}, \ldots, l_{p+q} ; k_{1}, \ldots, k_{p+q}\right) \\
& \prod_{i=1}^{p} D^{l_{i}} y\left(t-k_{i}\right) \prod_{i=p+1}^{p+q} D^{l_{i}} u\left(t-k_{i}\right)=0
\end{aligned}
$$

These three models all can get the GFRF. The advantage of the parameter identification method is that the arbitrary order of GFRF can be obtained by iterative calculation. The relationship between the non-linear term of time-domain mathematical model and GFRF can be obtained clearly. But its computational complexity is high. Recognition accuracy depends on the accuracy of the model, and it is limited in the practical application.

\section{Non-parametric identification method}

Non-parametric identification method is different from parameter identification method. It has no specific expression, which is according to the "black box" identification principle, don't need to know the internal mechanism and physical characteristics of the system, can identify GFRF based on input / output data only, and has the characteristics of fast and accurate .So it is more practical. At present, the main methods are timedomain adaptive identification method, the thirdorder simplified identification algorithm and the 
identification method based on the multi-tone signal.

\subsection{Time-domain adaptive identification method}

Time-domain adaptive identification method use adaptive identification method to solve the timedomain kernel. And then through the multidimensional Fourier transform to get the GFRF. The traditional method of adaptive identification in time-domain is based on the least square method (LSM).

The LSM of Volterra series model is expressed in vector form [7]:

$$
\vec{H}(\mathrm{k}+1)=\vec{H}(\mathrm{k})+\mu \vec{e}(k) \vec{U}(k)
$$

Where $\vec{e}(k)=\vec{d}(k)-\vec{H}^{T}(\mathrm{k}) \vec{U}(k)$ is residual , $\vec{d}(k)$ is the expected output of series model , $\vec{H}(\mathrm{k})$ 和 $\vec{U}(k)$ are the kernel vectors and input vectors of Volterra system , $\mu$ is learning factor, which controls the stability and the convergence speed of the adaptive identification algorithm.

However, LMS has some problems such as slow convergence speed. Scholars continue to optimize it. Ref. [8] designs the new LSM with high efficiency by using pseudo random multilevel sequence excitation, the inverse of the data matrix is avoided, the calculation efficiency and precision are improved; Ref. [9] proposes the LSM by fully-decoupled, the convergence speed and precision of the filtering process are improved, the adaptability of the filter to strong interference is improved. Ref. [10] uses recursive least squares method to identify the time-domain kernel, the results show that the method has high precision, fast convergence speed and better ability to resist noise. However, these algorithms require that the objective function can be continuously differentiable, is sensitive to measurement noise and need to use gradient information. With the development of intelligent algorithm, it provides a new idea for the kernel identification method [11]. Lin Haijun proposes the annealing genetic hybrid optimization algorithm, it has a good effect on the selection and extraction of fault features, and improves the accuracy of measurement; Li Zhinong has proposed a Volterra series identification method based on adaptive ant colony optimization and quantum particle swarm optimization, using the algorithm to estimate the kernel function of nonlinear system, the results show that the proposed method has better identification accuracy and convergence than traditional methods $[12,13]$.

The adaptive identification algorithm has the characteristics of small computation and high precision, however, because of it identifies the time-domain first, so the proposed method has the disadvantages of the time-domain kernel identification mentioned above, such as: strict requirements on the excitation signal, need to clear the memory length, which limit the application of this method.

\subsection{Third-order simplified identification algorithm}

Aiming to solve the problem of excessive identification parameters and curse of dimensionality, a very simple three-order simplified identification method is proposed. It is based on four assumptions: First, the dynamic behavior of non-linear system can be approximately described by the three-order Volterra kernel; Second, the system input signal is a linear combination of sine signal, fundamental frequency is $\omega_{0}$, other frequencies are integer multiples of the fundamental frequency, the cut-off frequency of input signal is $N_{r} \omega_{0}$; Third, the input and output signal are synchronous sampling; Fourth, it is assumed that the $\mathrm{n}$ th order Volterra is in the frequencydomain of the super plane, the value of GFRF is equal.

Third-order simplified identification algorithm steps are simple and easy to implement, however, it does not satisfy the assumption that the entire hyperplane is equal. And in practice, it does not satisfy the assumption that $H_{n}\left(\omega_{1}, \omega_{2}, \ldots, \omega_{n}\right)$ is equal to $\omega_{1}+\omega_{2}+\ldots+\omega_{k}=\omega$ over the entire hyperplane. Moreover, most non-linear systems can't be expressed only by the threeorder Volterra series, so the precision of the algorithm is limited.

\subsection{Identification method based on multi-tone signal}

Multi-tone excitation signal refers to the use of different frequency sine signal composed of multi tone signal as input signal. Through the analysis of GFRF model, we know that GFRF contains different frequency output components. In linear system, the frequency of the input signal is consistent with the output signal frequency. In non-linear systems, the output signal generates a new frequency component. According to the frequency components of the system output 
reverse GFRF, so as to realize the identification of GFRF [14].

Multi-tone signal can be expressed as [7]:

$$
\mathrm{u}(t)=\sum_{i=1}^{K} 2 a_{i} \cos \left(\omega_{i} t+\theta_{i}\right)=\sum_{\substack{i=-K \\ i \neq 0}}^{K} A_{i} e^{j \omega_{i} t}
$$

Where $A_{i}=\left|A_{i}\right| e^{j \theta_{i}}, A_{i}^{*}$ is the conjugate complex of $A_{i}$, and $A_{-i} \triangleq A_{i}^{*}, \omega_{-i} \triangleq-\omega_{i}$ 。

The frequency component contained in the multitone signal is $\omega_{1}, \omega_{2}, \ldots, \omega_{k}$, The first order nuclear output contains the frequency component $\left\{\omega_{i} \mid i=1,2, \ldots, K\right\}$; The second order nuclear output contains the frequency component $\left\{2 \omega_{i},\left|\omega_{i} \pm \omega_{j}\right| \mid i, j=1,2, \ldots, K ; \mathrm{i} \neq \mathrm{j}\right\}$, and so on. But the low order kernel output will be covered by the higher order kernel output. At present, the most effective way to separate the kernel is Vandermonde method, no more details here.

The frequency-domain identification method based on multi-tone signal also has the error. Jiao Licheng puts forward a fast multi point method for this problem [15]; Han Haitao introduced the correction factor in the frequency-domain kernel identification formula, the identification accuracy is improved [16]. Then optimizes the phase based on the selection of the frequency components of the multi-tone excitation signal, put forward a kind of phase optimization method based on genetic algorithm, which can effectively reduce the crest factor, ensure the signal power spectrum distortion [17]. Some scholars began to use intelligent algorithms to optimize the frequency of the excitation signal. Ref. [18] uses the ant colony algorithm to test the excitation optimization and achieves good effect.

The multi-tone excitation signal is used based in the multi tone signal identification method, which doesn't require knowledge of complex signal processing, and has the advantages of small calculation quantity, good anti-noise performance and easy realization, has good research prospect. However, there is no good solution to the problem of how to determine the memory length and the characteristics of the input signal quickly and accurately,

\section{Research prospect}

In the method of frequency-domain identification, parameter identification need accurate mathematical model. However, most of the nonlinear systems are complex in reality. Thus the parameter identification method based on the principle of "black box" has more research value and significance.

(1)There are many method in frequency-domain identification of Volterra series based on adaptive algorithm. At the same time, the development of Volterra series time-domain kernel identification will be also promoted and developed.

(2) In the identification method based on multitone excitation, the selection of input excitation signal is particularly important. Select the appropriate frequency and phase of the excitation signal, get the most useful information from the Volterra kernel, can achieve a multiplier effect. At present, the intelligent optimization algorithm is not mature, which can select the measure the maximum, and the dimension of feature vector can be controlled in a reasonable range; there is less research on the phase of the input signal; how to reduce errors in high order frequency-domain kernel identification, improve identification accuracy. These are the direction of future research.

\section{Conclusion}

With the development of computer technology, Volterra series shows its application value and great potential. The study of the frequencydomain identification method is also very important. In this paper, the Volterra series frequency-domain kernel identification method is introduced from two aspects of parameter identification and Non-parametric identification, and the future research directions are pointed out.

\section{Acknowledgements}

This work was supported by National Natural Science Foundation of China (Grant no. 61372039).

\section{References}

[1] Zhu A, Brazil T. J. Behavioral modeling of RF power amplifiers based on pruned Volterra series[J]. IEEE Microwave and Wireless Components Letters, 14, pp. 563-565, (2004)

[2] Kerschen Gaetan, Worden Keith, Vakakis Alexander F. et al. Past, present and future of nonlinear system identification in structural dynamics[J]. Mechanical Systems and Signal Processing, 20, pp. 505-592, (2016)

[3] He Jing, Zhao Zhong, Dong Yewei. Predictive control based on Volterra model and its 
application[J]. Control theory and Applications, 32, pp. 312-319, (2015)

[4] Avargel Y, Cohen I. Modeling and identification of non-linear systems in the shorttime Fourier transform domain[J]. IEEE Transactions on Signal Processing, 58, pp. 291304, (2010)

[5] Deng Yong, Shi Yibing, li Yanjun,et al. Soft fault diagnosis method for non-linear analog circuit[J]. Measurement and control technology, 32, pp. 22-25, (2013)

[6] Cao Jianfu, Han Chongzhao, Fang Yangwang. Theory and application of non-linear system[M]. Xi'an :Xi'an Jiao Tong University press, 2006

[7] Kong Yuxiang, Ma Hongguang, Han Chongzhao. Non-linear system modeling and fault diagnosis[M]. Beijing: Science Press, 2014.

[8] Toker O, Emara Shabaik H E. Pseudorandom multilevel sequences: Spectral properties and identification of Hammerstein systems[J]. IMA J Math Control I, 21, pp. 183-205, (2004)

[9] Wei Ruixuan, Han Chongzhao. A fully decoupled Volterra adaptive filter [J] Journal of Electronics, 6, pp. 839-841, (2001)

[10] Zhou Liqi. Research on fault diagnosis of non-linear analog circuits based on Volterra series [D]. Xi'an :Xi'an Electronic and Science University, 2012

[11] Lin Haijun, Fu Yao, Zhang Xuhui, $\mathrm{Xu}$ Zhicheng. The Study About Feature Selection of Analog Circuit Fault Diagnosis Based on Annealing
Genetic Hybrid Algorithm[C]. IFOST 2012, IEEE: $1-4$.

[12] Li Zhinong, Tang Gaosong, Xiao Xiaoxian, et al. Research on Volterra kernel identification algorithm based on adaptive ant colony optimization.[J] Vibration and Shock, 30, pp. :35$38,(2011)$

[13] Li Zhinong, Jiang Jing, Qiu Jingang, et al. Research on Volterra kernel identification algorithm based on quantum behaved particle swarm optimization [J] Vibration and shock, 32, pp. 60-63, (2013)

[14] Han Haitao, Ma Hongguang, Han Kun,et al. Design of multi-tone excitation signal in Volterra frequency-domain kernel identification[J]. Journal of Engineering Design, 19 , pp. 123-127, (2012)

[15] Jiao Licheng. Theory and application of non-linear transfer function [M]. Xi'an :Xi'an Electronic and Science University, 1992

[16] Han Haitao, Ma Hongguang, Yu Ningyu,et al. Non-parametric identification of Volterra frequency-domain kernel based on multi tone excitation [J]. Journal of Southwest Jiao Tong University, 48, pp. 250-255, (2013)

[17] Han Haitao, Tao Lining. Design of multi tone excitation for Volterra kernel identification based on GA theory[J]. Computer Engineering and Design, 34, pp. 1393-1398, (2013)

[18] Lin Haijun, Wang Qigao, et. The study of test stimulus optimization of analog circuit based on AS-PSO hybrid algorithm[J]. Applied Mechanics and Materials Vols, pp. 303-306, (2013) 